\title{
Role of Computed Tomography Scan in the Evaluation of Pancreatic Lesions
}

\author{
Sharvari Shashikant Gulve ${ }^{1}$, Pratapsingh Hanumantsingh Parihar ${ }^{2}$, Rajasbala Pradeep Dhande ${ }^{3}$ \\ 1, 2, 3 Department of Radiodiagnosis, Jawaharlal Nehru Medical College, Datta Meghe \\ Institute of Medical Sciences, Sawangi (Meghe), Wardha, Maharashtra, India.
}

\section{ABSTRACT}

\section{BACKGROUND}

Pancreatic lesions range from inflammation to malignancy and are associated with high morbidity and mortality. Imaging pancreatic lesion is challenging as pancreas is located retroperitoneally and with close proximity to bowel and major blood vessels. Abdominal pain, vomiting, and nausea are commonly reported symptoms. They are associated with high morbidity. Multi detector computed tomography (MDCT) is the modality of choice for detecting pancreatic pathology. It is highly sensitive in detecting necrosis, pancreatitis, peripancreatic fluid collections, calcification, neoplasm, pancreatic enlargement, atrophy and cystic lesions of pancreas. Computed tomography (CT) scan has made it possible to identify and detect various benign and malignant pancreatic lesions.

\section{METHODS}

A prospective observational study of 180 patients with complaints suggestive of pancreatic disease was done based on clinical, laboratory and ultrasonography in the Department of Radiodiagnosis, Acharya Vinoba Bhave Rural Hospital, Datta Meghe Institute of Medical Sciences, Sawangi (Meghe). Siemens Somatom 16 slice CT scan machine was used. All 180 patients underwent plain and contrast enhanced CT scan.

\section{RESULTS}

Out of 180 study participants 105 (58.33 \%) were with acute pancreatitis followed by $43(23.89 \%)$ with chronic pancreatitis, 21 (11.67\%) with acute exacerbation of chronic pancreatitis, 10 (5.56\%) participants with carcinoma pancreas and 1 (0.56 $\%)$ participant with pancreatic injury participated in the study.

\section{CONCLUSIONS}

Contrast enhanced computed tomography (CECT) is an excellent diagnostic modality to stage severity of inflammatory process, staging of neoplastic lesions and traumatic injury. It is most accurate and affordable non-invasive imaging modality for the evaluation of pancreatic lesions. It is a standard investigation to identify and quantify distribution of various pancreatic lesions and also evaluates activeness and progression of disease. Thus, it helps in accurate diagnosis and characterization of lesion and in proper treatment of patients.

\section{KEY WORDS}

Pancreas, Imaging, Computed Tomography
Corresponding Author:

Dr. Sharvari Shashikant Gulve, G17, Radhikabai PG hostel, DMIMS

Campus, Sawangi (Meghe), Wardha, Maharashtra, India.

E-mail: sharvari23@yahoo.com

DOI: $10.14260 / \mathrm{jemds} / 2021 / 175$

How to Cite This Article:

Gulve SS, Parihar PH, Dhande RP. Role of computed tomography scan in the evaluation of pancreatic lesions. J Evolution Med Dent Sci 2021;10(11):819-824, DOI: $10.14260 /$ jemds/2021/175

Submission 09-11-2020,

Peer Review 19-01-2021,

Acceptance 27-01-2021,

Published 15-03-2021.

Copyright (c) 2021 Sharvari Shashikant Gulve et al. This is an open access article distributed under Creative Commons Attribution License [Attribution 4.0 International (CC BY 4.0)] 


\section{BACKGROUND}

Pancreas is a hidden organ and one of the last organs in the abdomen to be analysed by anatomists, physiologists, physicians, and surgeons. ${ }^{1} \mathrm{CT}$ of the pancreas began in the late 1970's with simultaneous publication by authors like Kreel, Stephens et al. and Haaga et al. in 1976.2 Computed tomography is the non-invasive modality. It makes the use of computed processed combination of many x-ray measurements taken from different angles. It is also known as computed axial tomography.,2,3 Imaging in pancreatic lesion is challenging as pancreas is located retroperitoneally and with close proximity to bowel and major blood vessels. ${ }^{4,5}$ Pancreatic lesions include a spectrum of disease ranging from inflammation and its complication to malignancy. ${ }^{6,7}$ Abdominal pain, vomiting nausea are commonly reported symptoms. Pancreatic lesions are associated with high morbidity and comorbidity. ${ }^{6}$ Multi-detector computed tomography scan is modality of choice for detecting pancreatic pathology. It is highly sensitive in detecting necrosis, pancreatitis, peripancreatic fluid collections, calcification, neoplasm, pancreatic enlargement, atrophy and cystic lesions of pancreas..$^{2,3}$ CT scan has made it possible to identify and detect various benign and malignant pancreatic lesions. ${ }^{2,3} \mathrm{CT}$ scan is popular for diagnosing the pancreatic pathology due its availability and easy interpretation of CT images by radiologist. CT has the benefit of high accuracy over ultrasound in pancreatic lesions. ${ }^{8}$ Radiological evaluation with CT scan has become very essential for diagnosing the pancreatic lesions and planning their proper treatment. So, CT became the method of choice in evaluating the pancreas, in detecting a pancreatic lesion, assessing its extent, defining its aetiology and planning proper treatment. 2,3

As CT scan is highly reliable, can be easily performed. It has advantage of accuracy and accessibility. ${ }^{2}$ So present study is done to evaluate role of computed tomography in various pancreatic lesions include the spectrum of disease ranging from inflammation and its complication to malignancy, their characteristic appearance, clinical, biochemical and histopathological correlations. ${ }^{4-7}$

Radiological evaluation with CT scan has become very essential for diagnosing the pancreatic lesions and planning their proper treatment.

\section{Objectives}

- To evaluate various pancreatic lesions on CT scan.

- To assess prognostic correlation of patient's outcome with modified computed tomography severity indices.

- To differentiate various pancreatic masses on CT scan and correlation with histopathology.

\section{METHODS}

A prospective observational study of 180 (157 male and 23 female) patients with complaints suggestive of pancreatic disease on clinical, laboratory and ultrasonography basis were carried in Department of Radiodiagnosis, Acharya Vinoba Bhave Rural Hospital, Datta Meghe Institute of Medical Sciences, Sawangi (Meghe). Study was conducted from July
2018 to June 2020 (24 months). Siemens Somatom 16 slice CT scan machine was used. All 180 patients underwent plain and contrast enhanced CT scan.

\section{Inclusion Criteria}

1. Patients with clinical suspicion of pancreatic disease.

2. Patients with suspected pancreatic lesion on ultrasonography.

3. Patients with deranged biochemical tests for pancreatic disease.

\section{Exclusion Criteria}

1. Postoperative patients.

2. Pregnant females presenting with complaints of pancreatic lesion.

3. Patients allergic to contrast media.

\section{Assessment of Severity}

Assessment of severity in acute pancreatitis was done by Mortele Modified (Mortele KJ) Computed Tomography Severity Index (CTSI) scoring.

\section{Mortele Modified CTSI Scoring 9}

Pancreatic Inflammation

1. Normal pancreas - 0 point

2. Intrinsic pancreatic abnormalities with or without inflammatory changes in peripancreatic fat - 2 points

3. Pancreatic or peripancreatic fluid collection or peripancreatic fat necrosis - 4 point

The presence and extent of necrosis in each case was scored from 0 - 4 as follows:

1. Necrosis absent- 0 points

2. $<30 \%$ necrosis- 2 points

3. $30 \%$ necrosis- 4 points

To the above score, 2 points were added for the presence of extra pancreatic findings.

The modified CTSI was calculated by summing these values and the total score was then categorised as:

1. Mild pancreatitis modified CTSI score 0 - 2

2. Moderate pancreatitis modified CTSI score 4 - 6

3. Severe pancreatitis modified CTSI score 8 - 10"

\section{Statistical Analysis}

All responses were tabulated using Microsoft Excel 2007 software. Data was analysed by using SPSS software version 26. Statistical tools used were percentages, chi square test and statistical tests of significance.

\section{RESULTS}

Out of 180 study participants, 105 (58.33\%) patients presented with acute pancreatitis followed by $43(23.89 \%)$ patients with chronic pancreatitis, $21(11.67 \%)$ patients with acute exacerbation of chronic pancreatitis, 10 (5.56\%) participants with carcinoma pancreas and 1 (0.56\%) participant with pancreatic trauma were considered for study. 


\begin{tabular}{|ccc|}
\hline Diagnosis & No. of Patients & Percentage \\
\hline Acute pancreatitis & 105 & 58.33 \\
Chronic pancreatitis & 43 & 23.89 \\
Acute exacerbation of & 21 & 11.67 \\
chronic pancreatitis & 10 & 5.56 \\
Carcinoma pancreas & 01 & 0.56 \\
Pancreatic injury (trauma) & $\mathbf{1 8 0}$ & $\mathbf{1 0 0 . 0 0}$ \\
\hline Total & Table 1. Distribution of Cases According to Diagnosis \\
\hline
\end{tabular}

\section{Acute Pancreatitis}

In acute pancreatitis $40(38.10 \%)$ cases were in age group of 31 - 40 years followed by 32 (30.48\%) cases in the age group of 21 - 30 years, $20(19.05 \%)$ patients were in the age group of 41 - 50 years. Thus, most of the patients were in the age group of 20 - 50 years. Out of 105 cases of acute pancreatitis $92(87.62 \%)$ were males and $13(12.38 \%)$ were females

In acute pancreatitis most common symptom was pain which was observed in $103(98.09 \%)$ cases followed by vomiting in 51 (48.58) cases, nausea in 46 (43.80\%) cases. Most common aetiological factor of acute pancreatitis was alcohol in 76 (72.38 \%) cases followed by cholelithiasis in 15 $(14.28 \%)$ cases, and smoking in 8 (7.60 \%) cases.

In acute pancreatitis bulky parenchyma was observed in $103(98.09 \%)$ cases followed by the heterogenous enhancement in 65 (61.90\%) cases, necrosis less than $30 \%$ in $18(17.14 \%)$ cases, necrosis more than $30 \%$ was observed in $1(0.95 \%)$ case and normal pancreatic parenchyma was observed in $2(1.9 \%)$ cases.

In acute pancreatitis most common complication observed was ascites in $41(39.04 \%)$ cases followed by bilateral pleural effusion in 34 (32.38\%) cases, pseudocyst in 17 (16.1\%) cases, left sided pleural effusion in 13 (12.3\%) cases, gastrointestinal involvement in 11 (10.47 \%) cases, thrombosis in $9(8.50 \%)$ cases, splenic infarct in $2(1.9 \%)$ cases and right pleural effusion, splenic rupture and hepatic infarct in $1(0.95 \%)$ case each. Haemorrhage was not seen any case of acute and chronic pancreatitis.

As per modified CTSI out of 105 cases of acute pancreatitis, $28(26.67 \%)$ cases were of mild acute pancreatitis, 58 (55.24 $\%)$ cases were moderate acute pancreatitis and 19 (18.10\%) were of severe acute pancreatitis. In acute pancreatitis fluid collection or fat necrosis was seen in $64(60.95 \%)$ cases, fat stranding in 38 (36.19\%) cases, presence of gas in 4 (3.80\%) cases and no changes seen in $2(1.9 \%)$ cases. In mild cases hospital stay was $\leq 7$ days in $26(92.86 \%)$ cases and $8-14$ days in $2(7.14 \%)$ cases. In majority of cases of moderate acute pancreatitis hospital stay was 8 - 14 days in 32 (55.17\%) cases followed by $\leq 7$ days in 20 (34.48\%) cases, in severe cases hospital stay was $\leq 7$ days in $3(15.79 \%)$ cases, $8-14$ days in $7(36.84 \%)$ cases, 15 - 21 days in $3(15.79 \%)$ cases, $22-28$ days in $2(10.53 \%)$ cases and $>28$ days in $4(21.05 \%)$ cases. Hospital stay was less in most of the cases of mild acute pancreatitis compared to moderate and severe cases and difference observed was statistically significant.

In acute pancreatitis, infection was present in 14 (13.33\%) cases. Infection was not observed in any case of mild pancreatitis. In moderate and severe cases of the acute pancreatitis infection was present in $2(3.45 \%)$ cases and 12 $(63.16 \%)$ cases respectively. Infection rate according to grades of acute pancreatitis was found to be statistically significant. In this study treatment of mild acute pancreatitis was conservative in all $28(100 \%)$ cases. In moderate pancreatitis treatment was conservative in 49 (84.48\%) cases, surgical in 7 (12.07 \%) cases and interventional radiology in 2 (3.45\%) cases. In severe pancreatitis treatment was conservative in 14 (73.68 \%) cases, surgical in 3 (15.79\%) and interventional radiology in 2 (10.53\%) cases. Management of patients according to grades of acute pancreatitis was found to be statistically not significant. In mild, moderate and severe acute pancreatitis $28(100 \%)$ cases, 57 (98.28\%) cases and $14(73.68 \%)$ cases were discharged respectively. In moderate and severe acute pancreatitis death was reported in $1(1.72 \%)$ and $5(26.32 \%)$ cases respectively. Outcome of patients according to grades of acute pancreatitis was found to be statistically significant.

In acute pancreatitis amylase and lipase was increased in $93(88.57 \%)$ and 96 (91.42\%) cases respectively.

\section{Chronic Pancreatitis}

In our study we found 43 patients of chronic pancreatitis. Most commonly affected age group was 31 - 40 years of age in 12 (27.91) patients, followed by 41 - 50 years in 11 (25.58) patients. Males were more affected than females. Alcoholism was most common aetiological factor found.

Most common symptom among cases with chronic pancreatitis was pain in 34 (79.06\%) cases followed by vomiting in 7 (16.27\%) cases.

In the present study in chronic pancreatitis main pancreatic duct dilatation was observed in 26 (60.46\%) cases followed by atrophic parenchyma in $22(51.16 \%)$ cases, and calcification in pancreas observed in18 (41.86 \%) cases. There was diffuse involvement of pancreas in all the cases of chronic pancreatitis.

In chronic pancreatitis serum amylase was decreased in 3 (7.3\%) patients and serum lipase was decreased in $1(2.43 \%)$ patient and serum amylase and lipase were increased only in 1 case $(2.3 \%)$.

\begin{tabular}{|c|c|c|c|c|c|c|c|c|c|c|c|c|}
\hline \multirow[t]{2}{*}{ Age Group } & \multicolumn{2}{|c|}{ Acute Pancreatitis } & \multicolumn{2}{|c|}{$\begin{array}{c}\text { Chronic } \\
\text { Pancreatitis }\end{array}$} & \multicolumn{2}{|c|}{ Acute on Chronic Pancreatitis } & \multicolumn{2}{|c|}{ Trauma } & \multicolumn{2}{|c|}{ Neoplasm } & \multicolumn{2}{|c|}{ Total } \\
\hline & Cases & $\%$ & Cases & $\%$ & Cases & $\%$ & Cases & $\%$ & Cases & $\%$ & Cases & $\%$ \\
\hline $0-10$ & 0 & 0.00 & 0 & 0.00 & 0 & 0.00 & 1 & 100 & 0 & 0.00 & 1 & 0.56 \\
\hline $11-20$ & 2 & 1.90 & 2 & 4.65 & 0 & 0.00 & 0 & 0.00 & 0 & 0.00 & 4 & 2.22 \\
\hline $21-30$ & 32 & 30.48 & 6 & 13.95 & 5 & 23.80 & 0 & 0.00 & 0 & 0.00 & 43 & 23.89 \\
\hline $31-40$ & 40 & 38.10 & 12 & 27.91 & 7 & 33.34 & 0 & 0.00 & 0 & 0 & 59 & 32.78 \\
\hline $41-50$ & 20 & 19.05 & 11 & 25.58 & 5 & 23.80 & 0 & 0.00 & 1 & 10 & 37 & 20.56 \\
\hline $51-60$ & 5 & 4.76 & 6 & 13.95 & 3 & 14.29 & 0 & 0.00 & 5 & 50 & 19 & 10.56 \\
\hline $60-70$ & 3 & 2.86 & 4 & 9.30 & 1 & 4.77 & 0 & 0.00 & 3 & 30 & 11 & 6.11 \\
\hline$>70$ & 3 & 2.86 & 2 & 4.65 & 0 & 00 & 0 & 0.00 & 1 & 10 & 6 & 3.33 \\
\hline Total & 105 & 100 & 43 & 100 & 21 & 100 & 1 & 100 & 10 & 100 & 180 & 100 \\
\hline \multicolumn{13}{|c|}{ Table 2. Distribution of Cases According to Age Group } \\
\hline
\end{tabular}




\begin{tabular}{|cccccccc|}
\hline Cases & \multicolumn{2}{c}{ Male } & \multicolumn{2}{c}{ Female } & \multicolumn{2}{c|}{ Total } \\
& No. of Cases & \% & No. of Cases & \% & No. of Cases & $\%$ \\
\hline Acute pancreatitis & 92 & 87.62 & 13 & 12.38 & 105 & 100 \\
Chronic pancreatitis & 41 & 95.35 & 2 & 4.65 & 43 & 100 \\
$\begin{array}{c}\text { Acute on chronic } \\
\text { pancreatitis }\end{array}$ & 19 & 90.48 & 2 & 9.52 & 21 & 100 \\
$\begin{array}{c}\text { Pancreatic trauma } \\
\text { Neoplasm }\end{array}$ & 1 & 100 & 0 & 0 & 1 & 100 \\
\hline Total & 4 & 40.00 & 6 & 60.00 & 10 & 100 \\
\hline \multicolumn{6}{|c|}{ Table 3. Distribution of Cases According to Gender } \\
\hline
\end{tabular}

\section{Acute Exacerbation of Chronic Pancreatitis}

In present study acute exacerbation of chronic pancreatitis was observed in 21 cases and similar findings were not discussed in other study separately. The most common age group affected was 31 - 40 years of age in 7 patients (33.34\%), followed by 21 - 30 years and 40 - 50 years age group in 5 patients (23.8\%) each. Males were affected more than females. Alcoholism was most common aetiological factor followed by cholelithiasis.

The CT finding in acute exacerbation of chronic pancreatitis include dilated main pancreatic duct in 20 (95.24) and atrophic pancreas in 19 (90.47 \%) patients. In peripancreatic changes, peripancreatic fat stranding was seen in 15 (71.43) patients and fluid collection was seen in 6 (28.57 $\%)$ patients. There was diffuse involvement of gland in all the cases of acute exacerbation of chronic pancreatitis.

Most common complication found was ascites in 8 (38) patients and pseudocyst in 7 (33.33) patients. Left sided pleural effusion was found in 4 (19.04) patients and bilateral pleural effusion was found in $2(9.52 \%)$ patients. Pseudoaneurysm, was found in 1 (4.7\%) patient.

We applied modified CTSI to acute exacerbation of chronic pancreatitis and found that $10(47.62 \%)$ were mild and 11 (52.38\%) were moderate as per Modified Computed Tomography Severity Index MCTSI grading.

In mild cases of acute on chronic pancreatitis hospital stay was $\leq 7$ days in $9(90 \%)$ cases and 8 - 14 days in $1(10 \%)$ cases. In moderate cases hospital stay was $\leq 7$ days in 4 (36.36 $\%$ ) cases, 8 - 14 days in 4 (36.36\%) cases and 15 - 21 days in $3(37.28 \%)$ cases. Hospital stay was less in most of the cases of mild acute on chronic pancreatitis compared to moderate cases of acute on chronic and difference observed was not statistically significant.

Infection was absent in all the mild cases of acute on chronic pancreatitis. In moderate cases of acute on chronic pancreatitis infection was present in 2 (18.18\%) cases. Presence of infection according to grades of acute on chronic pancreatitis was found to be statistically not significant.

In mild cases of acute exacerbation of chronic pancreatitis treatment was conservative in all 10 (100\%) cases. In moderate cases of acute on chronic pancreatitis treatment was conservative in $10(90.91 \%)$ cases and radiological interventional in $1(9.09 \%)$ case. Management of patients according to grades of acute on chronic pancreatitis was found to be statistically not significant.

In mild and moderate acute on chronic pancreatitis all 10 (100\%) cases and 10 (90.91\%) cases were discharged respectively. In moderate acute on chronic pancreatitis death was reported in 1 (9.09\%) case. Outcome of patients according to grades of acute on chronic pancreatitis was found to be statistically not significant.

\section{Pancreatic Trauma}

In our study we had 1 case of pancreatic laceration i.e. grade II pancreatic injury according to AAST grading. It was a 10-year old boy who came with a complaint of abdominal pain after blunt trauma to abdomen. There was laceration at the junction of body and tail of pancreas. The patient had no signs of infection and was managed conservatively. Serum amylase and lipase were raised in this case.

\section{Neoplasm}

In the present study there were 10 patients of carcinoma pancreas. The most common pancreatic neoplasm was adenocarcinoma of pancreas. In the present study all the cases with neoplasm of pancreas was above the age of 50 years. In this study females were affected more than males. Pain, decreased appetite and weight loss were the common complaints found.

In neoplasms of pancreas, location was head, neck and uncinate process in $9(60 \%)$ cases followed by body and tail in $3(20 \%)$ cases each.

Neoplasm was localised in $3(30 \%)$ cases, invasion in adjacent structure in 3 (30\%) cases, lymphadenopathy was seen in $2(20 \%)$ cases and metastasis were seen in $6(60 \%)$ cases. In pancreatic neoplasm CA-19 and CA-125 was increased in 3 (42.86) and 4 (57.14) cases respectively. Hospital stay, death rate and rate of infection is as described in Table 1.

\section{DISCUSSION}

A total of 180 patients from outpatient department (OPD) and inpatient department (IPD) were included in present study to assess the role of computed tomography in evaluation of pancreatic lesion.

Abhijit Patil et al. ${ }^{10}$ in his study observed that commonest pancreatic pathology was acute pancreatitis followed by chronic pancreatitis and pancreatic carcinoma respectively. Riya Jeeson et al. ${ }^{11}$ had included 50 cases in the study and observed that 19 patients $(\sim 38 \%)$ had acute pancreatitis, 25 patients $(\sim 50 \%)$ had chronic pancreatitis and neoplasm was observed in 6 patients $(\sim 12 \%)$.

\section{Acute Pancreatitis}

Abhijit Patil et al. ${ }^{10}$ observed that in acute pancreatitis most of patients were in the middle age group of 20 - 50 years, similar to present study. He also observed that males are commonly affected than females in acute pancreatitis.

Yamandeep Chauhan et al. ${ }^{12}$ observed that the majority of patients of acute pancreatitis had complaint of abdominal pain (100.0\% cases), followed by nausea and vomiting in $42 \%$ cases.

Abhijit Patil et al. ${ }^{10}$ observed that alcohol (70 \%) is commonest etiological factor for acute pancreatitis followed by cholelithiasis ( $9 \%$ ) and trauma (4\%) was least causative factor which were similar to our findings. He also observed that diffuse enlargement in $61 \%$, regular contour in $61 \%$, homogenous density in $70 \%$ cases of acute pancreatitis. 
Michael C. Hill et al. ${ }^{13}$ also observed that maximum patients showed diffuse enlargement of gland in acute pancreatitis.

Pallavi Agrawal et al. ${ }^{14}$ observed that fat necrosis within and peripancreatic area in 14 (33\%) cases and haemorrhage was seen in $5(12 \%)$ cases of acute pancreatitis.

In his study Abhijit Patil et al. ${ }^{10}$ observed that peri peripancreatic fluid accumulation in $48 \%$ cases of acute pancreatitis. Riya Jeeson et al. ${ }^{11}$ found that out of 19 cases of acute pancreatitis, bulky pancreas were observed in 13 patients (68 percent), peripancreatic stranding was seen in 18 patients ( $~ 94$ percent), peripancreatic fluid accumulation was observed in 11 cases (57.8 percent) and pancreatic necrosis was seen in 6 patients ( 31.5 percent). Manu R et al. ${ }^{15}$ in the study observed pseudocyst in $15(30 \%)$ cases of acute pancreatitis.

Abhijit Patil et al.10 in his study observed that pseudocyst was present in $48 \%$ cases, ascites in $50 \%$ cases and pleural effusion in $48 \%$ cases of acute pancreatitis. Santh Kumar Bellamkonda et al. ${ }^{16}$ observed that pleural effusion on left side in $10 \%$ cases, pleural effusion in right lung only in $3.3 \%$ patients, bilateral pleural effusion in $3.3 \%$ patients of acute pancreatitis, thrombosis was seen in $3.3 \%$ cases and haemorrhage was not seen in any case, which is similar to the observation in the our study.

Among the complications seen in acute pancreatitis, Riya Jeeson et al. ${ }^{11}$ found that pleural effusion was more frequent and seen in 8 patients (42.2\%), accompanied by ascites in 7 patients (37\%), vascular complications were observed in 3 patients, among whom vascular thrombosis was more common and seen in 2 patients (10.4\%), and pseudoaneurysm in only 1 case $(5.2 \%)$. Manu R et al. ${ }^{15}$ observed that as per MCTSI score 3 (6\%) cases were mild, 35 (70\%) cases were moderate and $12(24 \%)$ cases were of severe acute pancreatitis. Riya Jeeson et al. ${ }^{11}$ observed that when modified CT Severity Index was employed, acute pancreatitis was graded as mild in $42 \%$ cases, moderate in $53 \%$ cases and severe in $5 \%$ patients.

Shalabh Jain et al. ${ }^{17}$ observed that the average span of hospital stay in the mild cases was 4.8 days, in moderate grade cases was 7.3 days and in severe cases was 13.5 days.

Santh Kumar Bellamkonda et al. ${ }^{16}$ observed infection in 10 (30\%) patients.

Shalabh Jain et al. ${ }^{17}$ observed that $30(20 \%)$ patients with acute pancreatitis had evidence of infection. He also observed that infection developed in $10 \%$ cases of moderate and $90 \%$ cases of acute severe pancreatitis.

Santh Kumar Bellamkonda et al. ${ }^{16}$ observed that a total of $7(23.3 \%)$ patients with acute pancreatitis underwent either percutaneous or surgical interventions. Shalabh Jain et al. ${ }^{17}$ showed that $30(20 \%)$ patients with acute pancreatitis underwent either percutaneous or surgical intervention. He also observed that $80 \%$ patients with severe acute pancreatitis and $20 \%$ patients with moderate acute pancreatitis required interventional treatment.

In acute pancreatitis out of 105 cases, 99 (94.29\%) were discharged and 6 (5.71 \%) patients died. Santh Kumar Bellamkonda et al. ${ }^{16}$ observed overall mortality rate of $3.3 \%$ in his study. Negi et al.. ${ }^{18}$ showed recovery of $94.3 \%$ of the patients with $5.7 \%$ of the mortality, which is similar to the observations in the present study.

Manu $\mathrm{R}$ et al. ${ }^{15}$ observed that amylase was elevated in 43 (86\%) patients at presentation and lipase was elevated in 45
(90\%) patients. Aastha Bhatt et al. ${ }^{19}$ observed that serum amylase was raised in 25 (50\%) patients of acute pancreatitis.

\section{Chronic Pancreatitis}

Riya Jeeson et al.11 found that 19 patients (76 percent) had pancreatic atrophy and main pancreatic duct dilatation, 20 patients (80 percent) had pancreatic parenchymal and intraductal calcification, and 13 patients (50 percent) had pseudocysts of chronic pancreatitis. Joseph T. Ferrucci et al. ${ }^{20}$ observed parenchymal atrophy in $14 \%$ cases, calcification in $36 \%$ cases and pancreatic duct dilatation in $4 \%$ cases of chronic pancreatitis.

Abhijit Patil et al. ${ }^{10}$ observed in chronic pancreatitis that pancreatic ductal dilatation (71\%), parenchyma calcification (62\%), alteration in peri-pancreatic fat (57 \%) were commonest signs.

Kathleen Barry ${ }^{21}$ showed incidence of pseudocyst in 25 $30 \%$ patients with chronic pancreatitis. Joseph T. Ferrucci et al. ${ }^{20}$ also showed that pseudocyst in $25 \%$ cases of chronic pancreatitis. Similar findings were observed in this study where pseudocyst was observed in 15 (34.88\%) cases

\section{Neoplasm of Pancreas}

Mahmoud Abdelaziz Dawoud et al.22 observed pancreatic mass in $70 \%$ cases above the age of 50 years. Soumail Singhal ${ }^{23}$ in his study observed that majority of the patients were above 50 years of age and the females were more affected than males

Partric Freeny et al. ${ }^{24}$ also showed in neoplasm of pancreas, pancreatic mass in $96 \%$ patients with the mass being focal in $95 \%$ and diffuse in $5 \%$; Of the focal masses $(62$ $\%)$ maximum were in the pancreatic head. Riya Jeeson et al. $^{3}$ noted that 3 of the 6 cases of pancreatic neoplasms had neoplasm located in the pancreatic head and uncinated phase (50\%) and 1 neoplasm located in the body and 2 cases of neoplasm located in the pancreatic tail region.

Riya Jeeson et al. ${ }^{11}$ noted locoregional lymphadenopathy in 4 patients (67 percent). In 4 patients (66.67 percent), distant metastases were seen and in 2 (33.33 percent) patients, involvement of adjacent organs and vascular involvement were seen.

\section{CONCLUSIONS}

CECT is excellent diagnostic modality to assess the severity of inflammatory process, traumatic injury and neoplastic lesions. Severity grading in acute exacerbation of chronic pancreatitis was meticulously observed and significant conclusive findings were found. CECT imaging with its postprocessing techniques is the imaging modality of choice for diagnosing pancreatic masses. It is a standard investigation to identify and quantify distribution of various pancreatic lesions and also evaluates activeness and progression of disease. It helps in accurate diagnosis and characterization of lesion and in proper treatment of patients.

Data sharing statement provided by the authors is available with the full text of this article at jemds.com.

Financial or other competing interests: None. 
Disclosure forms provided by the authors are available with the full text of this article at jemds.com.

\section{REFERENCES}

[1] Busnardo AC, DiDio LJ, Tidrick RT, et al. History of the pancreas. Am J Surg 1983;146(5):539-50.

[2] Haaga JR, Dogra V, Forsting M, et al. CT and MR imaging of the whole body. $5^{\text {th }}$ edn. Philadelphia: Elsevier 2003: 1599-667.

[3] Chaudhary V, Bano S. Imaging of the pancreas: recent advances. Indian J Endocrinol Metab 2011;15(Suppl1):S25-32.

[4] Moo TA, Zarnegar R, Brunaud L. Pancreas: embryology, anatomy and physiology. Endocrine Surgery 2009: p. 45969.

[5] Magee DJ, Burdick JS. Anatomy, histology, embryology and development anomalies of the pancreas. In: Feldman M, Friedman LS, Sleisenger MH, eds. Gastrointestinal and liver diseases: pathophysiology, diagnosis and management. $7^{\text {th }}$ edn. Philadelphia: W.B. Saunders Co, 2002: p. 859-70.

[6] Nealon WH. Surgical diseases of the pancreas. Ann Surg 2001;233(4):595-6.

[7] Vaughn DD, Jabra AA, Fishman EK. Pancreatic disease in children and young adults: evaluation with CT. Radiographics 1998;18(5):1171-87.

[8] Lee JK, Stanley RJ, Melson GL, et al. Pancreatic imaging by ultrasound and computed tomography. Radiol Clin North Am 1979;17(1):105-17.

[9] Mortele KJ, Wiesner W, Intriere L, et al. A modified CT severity index for evaluating acute pancreatitis: improved correlation with patient outcome. AJR Am J Roentgenol 2004;183(5):1261-5.

[10] Patil A, Valekar SV, Deshmukh A, et al. Role of CT scan in evaluation of pancreatic lesions. Sch J App Med Sci 2018;6(5):2248-52.

[11] Jeeson R, Balachandran A. Role of multidetector computed tomography in the characterization of pancreatic lesions. J Evid Based Med Healthc 2016;3(91):4991-5000.

[12] Chauhan Y, Jindal N, Verma RK, et al. A clinical profile and outcome of patients with acute pancreatitis: a prospective study in North India. Arch Int Surg 2018;8(3):132-8.
[13] Hill MC, Barkin J, Isikoff MB, et al. Acute pancreatitis: clinical vs. CT findings. AJR Am J Roentgenol 1982;139(2):263-9.

[14] Agrawal P, Vaiphei K. Histomorphological features of pancreas and liver in chronic alcoholics--an analytical study in 390 autopsy cases. Indian J Pathol Microbiol 2014;57(1):2-8.

[15] Manu R, Kumar MRS, Nanjarajand CP, et al. CT evaluation of acute pancreatitis and its complications using modified CT severity index. Int J Adv Res 2020;8(06):1266-312.

[16] Bellamkonda SK, Koneru VV, Ramadugu A, et al. Evaluation of modified computed tomography severity index in acute pancreatitis. International Journal of Anatomy, Radiology and Surgery 2020;9(1):R006-9.

[17] Jain S, Gupta S, Chawla AS, et al. Comparative study of balthazar computed tomography severity index and modified computed tomography severity index in predicting the outcome of acute pancreatitis. Apollo Medicine 2014;11(12):74-83.

[18] Negi N, Mokta J, Sharma B, et al. Clinical profile and outcome of acute pancreatitis: a hospital-based prospective observational study in Subhimalayan State. J Assoc Physicians India 2018;66(3):22-4.

[19] Bhatt A, Tiparse A, Patel A, et al. USG and CT scan evaluation of patients of acute and chronic pancreatitis-a cross-sectional, comparative study. Int J Res Med Sci 2017;5(8):3713-6.

[20] Ferrucci JT, Wittenberg J, Black EB, et al. Computed body tomography in chronic pancreatitis. Radiology 1979;130(1):175-82.

[21] Barry K. Chronic pancreatitis: diagnosis and treatment. Am Fam Physician 2018;97(6):385-93.

[22] Dawoud MA, Youssef MA, Elbarbary AA. Role of multidetector computed tomography in the evaluation of pancreatic tumors. The Egyptian Journal of Radiology and Nuclear Medicine 2014;45(2):309-16.

[23] Singhal S, Prabhu NK, Sethi P, et al. Role of multi detector computed tomography (MDCT) in preoperative staging of pancreatic carcinoma. J Clin Diagn Res 2017;11(5):TC015.

[24] Freeny PC. Impacted bile duct stone and chronic pancreatitis. Am J Roentgenol 2001;176(4):898. 